\title{
Des systèmes de formation sous influence
}

Approche comparée du Cameroun et de la Guinée

Training systems under influence. A comparative approach to Cameroon and Guinea.

\section{Valèse Mapto Kengne}

\section{OpenEdition Journals}

Édition électronique

URL : http://journals.openedition.org/ries/2846

DOI : $10.4000 /$ ries.2846

ISSN : 2261-4265

Éditeur

Centre international d'études pédagogiques

\section{Édition imprimée}

Date de publication : 1 décembre 1998

Pagination : 135-142

ISSN : $1254-4590$

Référence électronique

Valèse Mapto Kengne, "Des systèmes de formation sous influence », Revue internationale d'éducation de Sèvres [En ligne], 20 | décembre 1998, mis en ligne le 17 avril 2013, consulté le 19 avril 2019. URL: http://journals.openedition.org/ries/2846 ; DOI : 10.4000/ries.2846

Ce document a été généré automatiquement le 19 avril 2019

(c) Tous droits réservés 


\title{
Des systèmes de formation sous influence
}

\author{
Approche comparée du Cameroun et de la Guinée \\ Training systems under influence. A comparative approach to Cameroon and \\ Guinea.
}

\section{Valèse Mapto Kengne}

1 Le siècle tire à sa fin. L'Afrique est sinistrée. Certains systèmes éducatifs sont en crise. La formation des enseignants fait l'objet de débats et de recherches à l'échelle locale, nationale, régionale et internationale. Les enjeux sont énormes : il s'agit de former des citoyens éclairés, des enseignants compétents, pour des besoins et des demandes parfois mal définis.

2 Le regard se tourne vers les institutions de formation pédagogique, comme les écoles normales, les départements d'éducation ou les facultés des sciences de l'éducation pour interroger leurs raisons d'être. En effet, l'éducation est la clé du succès pour toute société digne et soucieuse de transmettre et de perpétuer des savoirs et des valeurs. Elle est le moteur du progrès social et humain. De ce fait, le problème est celui de rendre les acteurs $\mathrm{du}$ système éducatif responsables et autonomes. Autrement dit, il s'agit de professionnaliser le métier d'enseignant là où les besoins sont urgents.

3 Au Cameroun et en Guinée, les problèmes issus d'un héritage mal assumé rendent le débat obscur. Les multiples facteurs interdépendants qui conditionnent le développement de l'éducation sont ignorés. Les questions qui se rapportent aux langues nationales, aux minorités ethniques, au patrimoine culturel, à la participation des communautés et des parents à l'école ne sont pas prises en considération. Dans ces conditions, l'école ferme plus de portes qu'elle ne pourrait en ouvrir. De plus les différents cycles du système d'éducation ne s'articulent pas de manière à répondre aux besoins de ceux à qui ils s'adressent.

4 C'est à partir de cette constatation que nous avons cherché à voir si ces deux contextes culturels présentaient des différences ou des similitudes sur la problématique de la 
formation des enseignants du primaire et du secondaire. Le Cameroun est resté plutôt néo-libéral avec un système d'éducation inscrit dans un processus de francisation et la Guinée, socialiste, s'était inscrite dans un processus de rupture et de continuité avec le système français. En outre, de 1968 à 1984, la Guinée avait introduit l'enseignement de huit langues nationales à l'école. Sans méconnaître la nécessité d'une formation appropriée des enseignants au Cameroun et en Guinée, nous dresserons l'état des lieux à partir de trois questions : où et comment devient-on enseignant du primaire et du secondaire au Cameroun et en Guinée ? Quels sont les critères de recrutement et combien de temps consacre-t-on à la formation? Quels sont les facteurs qui favorisent et/ou inhibent le développement de la formation du personnel enseignant dans ces deux pays ? Sans vouloir généraliser la situation à toute l'Afrique, il s'agira d'envisager des perspectives d'ajustement.

\section{Le Cameroun et la Guinée}

Le Cameroun a été découvert au $\mathrm{XI}^{\mathrm{e}}$ siècle par les Portugais et passe sous protectorat allemand de 1884 à 1922. En 1922, il est placé sous mandat français et britannique. Le $1^{\mathrm{er}}$ janvier 1960, le Cameroun devient un État indépendant. La Guinée, colonie de langue française depuis 1893, rompt avec la France en 1958 pour tisser des liens avec l'URSS. Le système éducatif et le système de formation des enseignants sont influencés par les modèles français et anglais au Cameroun, français et soviétique en Guinée. Ces systèmes éducatifs sont centralisés.

6 Au Cameroun, c'est le ministère de l'Éducation nationale, en Guinée, le ministère de l'Éducation nationale et le ministère de l'Enseignement pré-universitaire qui réglementent et organisent les concours et les examens à l'échelle nationale, pour l'entrée dans les lycées, les collèges et les écoles de formation des enseignants. Les institutions de formation des maitres sont ouvertes dans plusieurs provinces, et les institutions s'adressant aux professeurs du secondaire ne se limitent qu'aux grands centres urbains, Conakry en Guinée, Yaoundé et Douala au Cameroun. L'éducation préscolaire n'est pas généralisée et la fréquentation scolaire obligatoire n'existe dans aucun des deux pays. Le système éducatif guinéen dans ses grandes lignes « recherche les voies et moyens susceptibles de résoudre efficacement les problèmes de développement " 1. Au Cameroun et en Guinée, la construction de l'unité nationale malgré les grandes disparités entre les régions du Nord, du Sud, de l'Est et de l'Ouest est au coeur des préoccupations du système éducatif. L'enseignement technique et professionnel vise la formation des ressources humaines. Dans cette optique, nous nous limiterons à la formation des enseignants du primaire et du secondaire de l'enseignement public.

7 Au Cameroun et en Guinée, l'école primaire comprend six classes, donc six années d'études. Le cycle s'achève par le certificat d'étude primaire et élémentaire (CEPE). Les maîtres du primaire sont formés à l'école normale d'instituteur ou d'instituteur-adjoint (ENI ou ENIA). L'enseignement secondaire comprend deux cycles et offre deux filières : l'enseignement général et l'enseignement technique ou professionnel. Sept classes en tout la composent et s'achèvent d'abord au bout de quatre ans par le BEPC ou le CAP. Ensuite, au terme de trois ans, l'élève se présente au baccalauréat. Au Cameroun, les enseignants du secondaire sortent de l'école normale supérieure (ENS) ou de l'école normale supérieure de l'enseignement technique (ENSET). En Guinée, ils sortent soit de 
l'école normale secondaire (ENSEC), soit de l'institut supérieur des sciences de l'éducation (ISSE) ou de l'école normale secondaire technique (ENSET).

\section{Les systèmes de formation}

\section{La formation dans les ENI/ENIA pour l'enseignement élémentaire}

8 Au Cameroun, le niveau du baccalauréat est exigé des étudiants de l'ENI et de l'ENIA. En Guinée, le brevet d'études du premier cycle secondaire (BEPC) est seul exigé à l'ENI avec neuf ans de scolarité à l'ENP, soit trois ans après le CEPE. La formation dans les deux pays dure deux à trois ans. Le diplôme des écoles permet de distinguer parmi les maîtres : les instituteurs ordinaires, les instituteurs adjoints et les moniteurs. La Guinée où $80 \%$ de la population est musulmane, forme en plus des instituteurs de langue arabe.

\section{La formation à l'ENS, I'ENSEC, l'ISSE, I'ENSET pour l'enseignement secondaire}

9 L'ENSET et l'ENS au Cameroun, l'ENSEC, l'ISSE et l'ENSET (Secondaire) en Guinée ne correspondent pas tout à fait. Cependant, ENS, ENSEC et ISSE préparent tous les professeurs de la filière générale des premier et deuxième cycles du secondaire: ENS et ENSEC pour le premier cycle (trois années de formation), ISSE pour le deuxième cycle (une année de formation).

$\mathrm{Au}$ Cameroun, l'ENS et l'ENSET comprennent deux cycles de formation. Le premier cycle de trois ans forme les professeurs du premier cycle du secondaire, titulaires du diplôme de professeur de collège d'enseignement général (DIPCEG) ou du diplôme de professeur d'enseignement technique I (DIPET-I). Le deuxième cycle de deux ans, prépare les professeurs du second cycle du secondaire au diplôme de professeur de lycée d'enseignement général (DIPLEG) ou de DIPET-II. L'ENS forme, en plus, les inspecteurs de l'enseignement primaire et les conseillers d'orientation.

11 Les candidats de l'ENSEC, de l'ENS et de l'ENSET premier cycle sont titulaires du baccalauréat et ceux de l'ISSE, de l'ENS et de l'ENSET second cycle sont titulaires d'une licence des facultés ou d'un diplôme des écoles de l'enseignement supérieur.

\section{Le contenu de la formation}

Les écoles de formation mettent l'accent sur les cours de pédagogie, de didactique, de psychologie de l'enfant, d'andragogie, de psychopédagogie, de techniques d'organisation et d'animation de groupe, de législation scolaire, de micro-enseignement, de sociologie et de philosophie de l'éducation.

La formation pratique est également de rigueur. Les étudiants ont une connaissance des disciplines ou des matières enseignées à l'école. Ces matières sont d'abord au primaire : la lecture, le dessin, l'écriture, le calcul, la science, l'histoire, la géographie, le chant et l'éducation physique. Ensuite au secondaire, on retrouve la couture, le travail manuel, la musique, le français, l'anglais, l'espagnol, l'allemand, l'histoire, la géographie, l'instruction civique, les sciences naturelles, la technologie, les mathématiques, la physique et la chimie, la philosophie, l'électricité, la mécanique, la comptabilité et le 
marketing. Les stages pratiques sont organisés dans les écoles et les lycées sous l'encadrement des enseignants titulaires qui sont évalués par un inspecteur national.

\section{Le statut des futurs enseignants}

$14 \mathrm{Au}$ Cameroun et en Guinée, les futurs enseignants sont des fonctionnaires qui seront à la disposition du ministère de l'Éducation nationale à la fin de leur formation.

15 Le ministère décide de leur affectation dans un établissement correspondant à leur filière et à leur niveau de formation. À l'ISSE de Guinée, les futurs professeurs reçoivent une allocation sous forme de bourse. Dans les autres établissements de formation, les futurs enseignants ne perçoivent pas d'allocation. Au Cameroun, depuis 1993, l'État camerounais a mis un terme au système d'allocation de bourses dans l'enseignement supérieur.

\section{Les facteurs affectant la formation}

\section{De rudes conditions de travail}

16 Les similitudes que nous pouvons constater entre les deux systèmes sont dues à l'influence du modèle français sur le développement du système éducatif. Le socialisme n'a pas modifié la structure du système guinéen. Cependant, on peut noter une rupture dans la durée de la formation et une faible exigence pour les candidatures des futurs enseignants. L'explication qu'on pourrait donner à cet état de chose est qu'historiquement la Guinée avait rompu ses liens avec la France pour l'ancienne URSS. L'économie des deux pays est en crise et cette situation a un impact considérable sur le développement de la formation du personnel enseignant. En effet, le PNB du Cameroun est de 820 \$ US, et le budget alloué à l'éducation représente 19,6\% de celui de l'État. La Guinée a 510 \$ US de PNB et 15,3\% du budget de l'État est alloué à l'éducation.

Le chômage des diplômés de l'enseignement supérieur a permis de résorber dans les grandes villes du Cameroun le problème de sous-qualification des enseignants. Ces derniers connaissent les matières à enseigner, mais n'ont reçu aucune formation en éducation, et plus particulièrement en pédagogie. Ils ne peuvent donc pas évaluer correctement les apprentissages des élèves, élaborer un plan de cours ni gérer une classe qui la plupart du temps a des effectifs pléthoriques. De plus, les élèves confiés n'appartiennent pas à la même classe d'âge. À ceci s'ajoutent les problèmes de communication, qui se situent à deux niveaux : d'abord au niveau du professeur et de ses élèves, ensuite au niveau de la transmission des contenus. En Guinée, le problème est plus urgent encore parce qu'il existe des enseignants du secondaire qui passent encore moins d'années dans les écoles de formation.

18 Malgré la présence des établissements de formation, le corps enseignant reste hétéroclite pour répondre au besoin du ministère de l'Éducation dans les deux pays. Plusieurs facteurs en interaction inhibent l'efficacité des enseignants et le développement de leur formation. Ce sont des facteurs en priorité d'ordre démographique, le problème des effectifs pléthoriques semble avoir un grand impact sur les systèmes de formation des enseignants. Les écoles de formation n'offrent pas les structures nécessaires pour augmenter les effectifs de futurs enseignants par promotion. Les écoles souffrent de la pénurie d'enseignants. Les comportements des élèves échappent aux enseignants et 
rendent leur tâche plus difficile. Au Cameroun, on compte 6709 écoles primaires, 1964146 d'élèves et 38430 enseignants. Ce qui porte le ratio maître/élève à 1/55. En Guinée, 471792 élèves sont encadrés par 9718 maîtres, dans 2849 écoles. Le ratio maître élève est de $1 / 50$. Il devient difficile d'enseigner dans des conditions où on peut atteindre des effectifs de 200 élèves par classe. À cette question d'effectif, s'ajoutent des conditions de travail assez rudes. Le manque de ressources matérielles, didactiques et pédagogiques, l'insalubrité des locaux, une image peu valorisée, des revenus faibles et une absence de structure syndicale font des enseignants des laissés pour compte.

Les enseignants ne sont pas consultés sur les différents ajustements apportés au système éducatif. Il serait opportun qu'ils aient un espace pour la défense de leur droit.

\section{Le poids des facteurs sociaux et culturels}

Les facteurs sociaux et culturels, tels que la non participation des parents à l'école, la non collaboration entre les enseignants plus qualifiés et les moins qualifiés, rendent l'encadrement des jeunes difficile.

21 Le facteur ethnolinguistique, la question des langues et des minorités ethniques représentent un réel enjeu pour les enseignants. Les programmes, dans les institutions, n'offrent ni des cours de langues nationales, ni des cours en langues nationales et encore moins en anthropologie. Pourtant, au Cameroun, cent quatre-vingt ethnies parlent plus de vingt langues. En Guinée, la situation n'est pas très différente. Il n'est pas toujours facile pour les enseignants de communiquer avec des élèves dont la langue de scolarisation constitue un véritable obstacle à la survie dans le système scolaire. Enseigner, c'est d'abord transmettre et perpétuer une tradition, un savoir et faire acquérir aux élèves des systèmes de signes et de représentation. Cette acquisition prend du temps et s'inscrit dans le long terme. Mais cette acquisition des signes n'a de sens que si elle passe par la langue naturelle, parce que la langue naturelle constitue le premier niveau de représentation. Dans ces pays, la formation des enseignants ne se préoccupe pas de cette situation, qui pourrait expliquer la pauvreté, l'abandon scolaire, l'analphabétisme et tous ses corollaires. Dans ce contexte, prétendre assurer le développement des élèves, des collectivités locales et de la modernisation dans le respect de l'autonomie, n'est qu'un leurre. Car, l'enseignant et l'élève dans certaines disciplines comme le français, l'histoire ou la géographie s'instruisent à partir des représentations qui ne correspondent pas a leur réalité. De surcroît, il faut avouer que les enseignants n'ont pas eux-mêmes souvent parfaitement assimilé la langue avec laquelle ils exercent leur métier. Au Cameroun et en Guinée, moins de $40 \%$ de la population parle les langues officielles. Au lieu de parler de francophone et anglophone, adjectifs attribuables seulement à l'élite, il est temps de fonder une bantouphonie pour récupérer les exclus du système d'éducation et construire une Afrique plus intégrée. Cela serait plus juste, en regard de l'exclusion et la discrimination que créent les langues étrangères.

Le facteur géographique et économique pose le problème de l'inégalité professionnelle entre les enseignants. Cela se traduit en termes de régions rurales et urbaines, de proximité des moyens de transport, de communication et d'industrie. Dans les régions urbaines, on assiste à la démotivation et au changement de carrière des enseignants. À cet égard, repenser la formation des maîtres en fonction des besoins des sociétés camerounaise et guinéenne devrait faire l'objet de projet de société. Il s'agit de mettre à contribution les nouvelles technologies de l'information pour pallier le manque de 
bibliothèques et de formation de niveau universitaire, d'encourager l'apprentissage tout au long de la vie et le multilinguisme (langues nationales et langues officielles) pour faciliter l'apprentissage des jeunes et réduire également les inégalités scolaires. Il faudrait créer des modules de formation sur le terrain pour les enseignants qui n'ont pas de qualification pédagogique et théorique suffisante.

\section{Héritage occidental et besoins de la société africaine}

Les problèmes auxquels la formation des enseignants se heurte au Cameroun et en Guinée, ne sont pas propres à ces deux pays. Plusieurs pays en Afrique vivent cette situation à des degrés variables. La disparité dans le corps enseignant nécessite que soit posé clairement le problème d'une formation adéquate des enseignants. Il s'agit de réfléchir sur les nouveaux fondements des systèmes éducatifs et de formation des enseignants en Afrique, d'établir de nouveaux rapports entre l'héritage occidental et les besoins des sociétés africaines. De toute évidence, la mission des systèmes d'éducation et de formation doit être en rapport avec la structuration de la société et ses besoins de conserver le patrimoine culturel, architectural et langagier des peuples en les portant vers le futur. Les systèmes de formation doivent aussi intégrer les valeurs, l'histoire et la langue des individus à qui elle s'adresse. Ces objectifs sont raisonnables et envisageables à long terme.

Pour conclure cette analyse, les écoles camerounaises et guinéennes, tout comme d'autres écoles en Afrique et dans le monde contemporain, doivent établir avec la société et l'environnement des relations effectives de deux ordres. D'abord une relation de transmission de valeur, voire de conservation du patrimoine en terme de langue, d'œuvres d'art, etc. Ceci n'est pas une réponse urgente à un besoin immédiat, c'est une transmission de longue durée qui se construit avec le temps. Elle place l'école au-dessus de tous les naufrages. La deuxième relation est celle de l'école du temps, qui ouvre ses portes aux marchés, à plusieurs types de partenariat pour enrichir l'humanité à travers les progrès accomplis par l'éducation. Ainsi, la synthèse du passé et du présent permettra d'anticiper sur le futur. Il serait nécessaire de viser, à travers un enseignement, la formation des individus capables de se remettre en question, d'être autonomes et responsables. Il s'agit de donner aux enseignants les moyens de transmettre des valeurs et une culture morale et scientifique, de former des hommes capables de résoudre des problèmes qui se poseront à eux dans un environnement donné. Face à la complexité des savoirs et aux mutations que le monde connaît actuellement, les enseignants doivent avoir plus de moyens dans leurs classes, pour répondre aux attentes des élèves. Or, nous constatons une tendance à la stagnation, au manque d'initiative et d'innovation, malgré les finalités raisonnables que les États camerounais et guinéen ont attribuées à leurs systèmes éducatifs. Cela pourrait conduire l'action éducative vers des problèmes encore plus complexes et insolubles à moyen terme. Le danger qui guette le Cameroun et la Guinée, c'est la dégradation des deux ordres d'enseignement que nous avons présentés cidessus. Pourtant, l'éducation est la base de tout système de promotion et de mobilité sociales. Il serait intéressant que les gouvernements investissent plus dans l'éducation et la formation des enseignants et que l'enseignement supérieur n'offre plus seulement ses chômeurs à l'éducation nationale, mais, qu'il entreprenne des recherches sociales et qualitatives sur les problématiques de l'éducation et, surtout, de la didactique de l'enseignement des langues nationales à l'école. C'est de cette manière que 
l'enseignement supérieur répondra efficacement aux enjeux de développement et de modernisation de ces deux pays. Les convergences qui existent entre le Cameroun et la Guinée, inspirent des changements essentiels dans la formation des enseignants autour de trois points :

- une participation des parents à la vie et à l'élaboration des savoirs transmis à l'école ;

- la flexibilité et l'innovation dans les contenus hérités de l'occident ;

- l'enseignement des langues et l'anthropologie culturelle dans les écoles de formation des enseignants.

- Nous ajouterons qu'il faut, dès l'école primaire, enseigner dans les langues nationales, en introduisant les langues étrangères et ensuite, aux niveaux secondaire et supérieur, donner des enseignements dans les langues étrangères.

En définitive, si selon Delors, «l'éducation : un trésor est caché dedans ", la clé du succès ou du trésor dépendra de la qualité des enseignants qui amèneront les jeunes Africains à découvrir ce trésor. Sans éducation ni formation d'enseignants de qualité, quelle sera la place de l'Afrique au XXI siècle?

\section{NOTES}

1. N. F. Barry, Une autopsie et l'entretien comme aides à la réflexion pédagogique auprès d'enseignants guinéens du professionnel en perfectionnement, Université de Sherbrooke, Faculté d'éducation, mémoire, 1995.

\section{RÉSUMÉS}

Comment dans deux contextes politiques et culturels différents, celui du Cameroun et celui de la Guinée, la formation des enseignants du primaire et du secondaire a-t-elle évolué ? Deux objectifs contradictoires semblent devoir être atteints : le respect de la société traditionnelle et l'entrée dans le XXI ${ }^{\mathrm{e}}$ siècle.

How has teacher training evolved, for primary and secondary school teachers, in two different political and cultural contexts, that of Cameroon and Guinea? It would appear that two contradictory aims have to be attained: respect for traditional society and entry into the twentyfirst century. 
INDEX

Mots-clés : enseignant, enseignement primaire, enseignement secondaire, formation des enseignants

Index géographique : Cameroun, Guinée

\section{AUTEUR}

VALÈSE MAPTO KENGNE

Doctorante en sciences de l'éducation, Université de Montréal, Canada 